\title{
The role of bilirubin to albumin ratio as a predictor for mortality in critically ill patients without existing liver or biliary tract disease
}

\author{
Ji Soo Choi, Kyung Soo Chung, Eun Hye Lee, Su Hwan Lee, Sang Hoon Lee, Song Yee Kim, Ji Ye Jung, \\ Young Ae Kang, Moo Suk Park, Young Sam Kim, Joon Chang, Ah Young Leem \\ Division of Pulmonology, Department of Internal Medicine, Severance Hospital, Yonsei University College of Medicine, Seoul, Korea
}

\begin{abstract}
Background: Hyperbilirubinemia and hypoalbuminemia are frequently appeared and associated with poor prognosis in critically ill patients. We aim to evaluate the association between the bilirubin to albumin ratio and prognosis in intensive care unit (ICU) patients.

Methods: This was a retrospective study of 731 patients who were admitted to the medical intensive care unit (MICU) at a tertiary-care center from July 2015 to September 2017. We analyzed the bilirubin to albumin ratio on admission to the MICU, including clinical characteristics and other examinations.

Results: The overall 28-day survival of MICU patients was 69.1\%. On univariate analysis, Acute Physiology and Chronic Health Evaluation (APACHE) II score $(\mathrm{P}<0.001)$, Sequential Organ Failure Assessment score $(\mathrm{P}<0.001)$, Simplified Acute Physiology Score II score $(\mathrm{P}<0.001)$, Creactive protein $(P=0.015)$, and bilirubin/albumin ratio $(P<0.001)$ were associated with mortality of ICU patients. The receiver operating characteristic curves for ICU patients mortality between bilirubin to albumin ratio and APACHE II score were not statistically significant $(\mathrm{P}=0.282)$. On multivariate analysis, higher APACHE II score (hazard ratio [HR], 1.05; 95\% Cl, 1.03 to $1.06 ; \mathrm{P}<0.001)$ and bilirubin to albumin ratio $(\mathrm{HR}, 1.65 ; 95 \% \mathrm{Cl}, 1.23$ to $2.20 ; \mathrm{P}=0.001)$ were independently related to the ICU patient mortality.

Conclusions: A higher bilirubin to albumin ratio was related to the unfavorable prognosis and mortality in critically ill patients.
\end{abstract}

Key Words: albumin; bilirubin; critically ill; mortality; prognosis

\section{INTRODUCTION}

Hepatic response plays an crucial role in the host defense mechanisms in sepsis or other critical illness patients [1-3]. Hepatic dysfunction is indicative of poor outcomes in critically ill patients [4], and abnormal liver function test, including elevated alanine aminotransferase (ALT), and aspartate aminotransferase (AST), alkaline phosphatase, and bilirubin, are traditionally presented in critically ill patients [5]. Hepatic dysfunction, particularly manifested as hyperbilirubinemia, is associated with poor outcomes [6,7]. Widely used scoring systems for sepsis, such as Sequential (sepsis-related) Organ Failure Assessment (SOFA) score and Simplified Acute Physiology Score (SAPS), included bilirubin level to estimate the hepatic dysfunction [8,9]. Low albu-

\section{Original Article}

Received: October 12, 2019

Revised: December 22, 2019

Accepted: January 30,2020

Corresponding author

Ah Young Leem

Division of Pulmonology, Department of Internal Medicine, Severance Hospital, Yonsei University College of Medicine, 50-1 Yonsei-ro, Seodaemun-gu, Seoul 03722, Korea Tel: $+82-2-2228-1991$ Fax: +82-2-393-6884 E-mail: yimayoung@yuhs.ac

Copyright $\odot 2020$ The Korean Society of Critical Care Medicine

This is an Open Access article distributed under the terms of Creative Attributions Non-Commercial License (https:// creativecommons.org/li-censes/by-nc/4.0/) which permits unrestricted noncommercial use, distribution, and reproduction in any medium, provided the original work is properly cited. 
min level is also helpful for predicting the poor prognosis in acutely ill patients $[4,10]$. Because of its importance for predicting the outcome in ICU patients, serum albumin level has included in the Acute Physiology and Chronic Health Evaluation (APACHE) III score [11].

Several studies of the relationship between serum bilirubin to albumin ratio (B/A ratio) and patient prognosis have been conducted $[12,13]$. In neonates, the $B / A$ ratio can be used to predict acute bilirubin-induced neurologic dysfunction [12,13]. However, there is no prior research about the relationship between the B/A ratio and prognosis in critically ill elderly patients. Therefore, we aim to evaluate if there is a relationship between the $\mathrm{B} / \mathrm{A}$ ratio and higher mortality in critically ill patients.

\section{MATERIALS AND METHODS}

\section{Patients and Study Design}

This was a retrospective cohort study of patients who admitted to the medical intensive care unit (MICU) at a tertiary-care university hospital in South Korea between July, 1, 2015 and September, 30, 2017. The medical ICU is a 30-bed closed unit where is managed by certified ICU specialists. A total of 867 patients were admitted to our MICU during study period. Among them, 136 patients who had the acute toxic or alcoholic hepatitis, chronic hepatitis, liver cirrhosis, cholecystitis with or without cholangitis, and those who had liver, biliary, or pancreatic surgery were excluded. A total of 731 patients were enrolled to this study (Figure 1).

The protocol was approved by institutional review board (IRB No. 4-2016-0951). The need for informed consent was waived given the retrospective nature of the study.

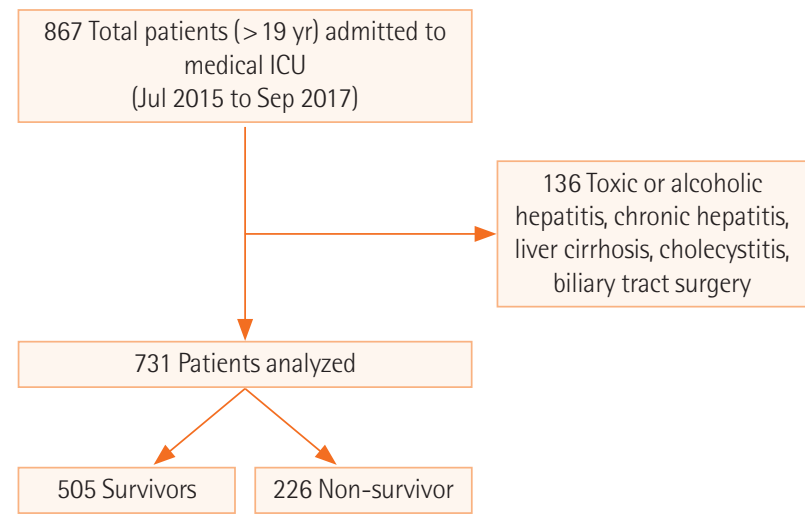

Figure 1. Flowchart of inclusion and exclusion process for patient enrollment. A total of 867 patients were enrolled, and 731 patients were included in the analysis. ICU: intensive care unit.

\section{KEY MESSAGES}

- Hepatic dysfunction is related to worse outcomes in critically ill patients.

- The purpose of this study is to analyze the role of the bilirubin to albumin ratio as a prognostic factor in critically ill patients.

\section{Data Collection}

The laboratory findings with serum bilirubin and albumin concentrations, including patients' baseline characteristics, disease severity such as APACHE II and SOFA scoring system, were reviewed to analyze the relevance to mortality in ICU patients. The laboratory tests used in this study conducted within 24 hours from admission of ICU, and disease severities were evaluated on ICU admission. The primary outcome was 28-day allcause mortality.

\section{Statistical Analysis}

The categorical variables are presented as absolute frequency and percentages, and the continuous variables are presented as mean \pm standard deviation. The clinical characteristics of the two groups, survivors and non-survivors, were compared using the Pearson's chi-squared test or Fisher's exact test for categorical variables and Student's t-test for continuous variables. Univariate logistic regression analysis was performed first, and $\mathrm{P}<0.05$ was considered statistically significant $[14,15]$. Multivariate analysis was performed using the forward stepwise data selection method $[14,15]$. Cutoff values of 0.05 and 0.1 were used to either enter or remove a covariate into or from the final model, and the results are described as odds ratio (ORs) with their 95\% confidence intervals (CIs). Cox proportional-hazard regression analysis was used to evaluate the association between variables and mortality. The effect of age, sex, body mass index (BMI) and APACHE II score was evaluated using Cox proportional-hazard models, with an adjusted P-value $<0.05$ considered statistically significant. The hazard ratios and 95\% CIs were calculated. Survival was estimated using the Kaplan-Meier method, and differences in survival between groups were assessed using the log-rank test. Anaylsis of receiver operating characteristic (ROC) curves and the area under the ROC curves (AUC) was performed to evaluate the predictive value of the $\mathrm{B} / \mathrm{A}$ ratio and APACHE II score for mortality in critically ill patients. In addition, we compared the ROC curves between $\mathrm{B} / \mathrm{A}$ ratio and APACHE II score. Kaplan-Meier analysis was used to evaluate the primary outcomes and P-values $<0.05$ were considered statistically significant. All statistical analyses were performed us- 
ing IBM SPSS ver. 23.0 (IBM Corp., Armonk, NY, USA) and MedCalc Statistical Software version 12.7.7 (MedCalc Software, Ostend, Belgium).

Table 1. Baseline characteristics of patients at ICU admission $(n=731)$

\begin{tabular}{|c|c|c|c|}
\hline Variable & $\begin{array}{l}\text { Survivor } \\
(n=505)\end{array}$ & $\begin{array}{l}\text { Non-survivor } \\
(n=226)\end{array}$ & P-value \\
\hline Age (yr) & $65.6 \pm 15.2$ & $67.9 \pm 13.1$ & 0.043 \\
\hline Male sex & $311(61.6)$ & $137(60.6)$ & 0.805 \\
\hline BMI $\left(\mathrm{kg} / \mathrm{m}^{2}\right)$ & $22.5 \pm 8.2$ & $22.1 \pm 5.1$ & 0.470 \\
\hline \multicolumn{4}{|l|}{ Disease severity } \\
\hline APACHE II score & $21.6 \pm 9.9$ & $27.5 \pm 10.5$ & $<0.001$ \\
\hline SOFA score & $7.7 \pm 3.9$ & $10.6 \pm 4.0$ & $<0.001$ \\
\hline SAPS II score $(n=484)$ & $37.9 \pm 16.3$ & $50.5 \pm 19.3$ & $<0.001$ \\
\hline \multicolumn{4}{|l|}{ Underlying disease } \\
\hline Diabetes mellitus & $189(37.4)$ & 73 (32.3) & 0.182 \\
\hline Chronic lung disease $^{a}$ & $89(17.6)$ & $35(15.5)$ & 0.477 \\
\hline Hypertension & $281(55.6)$ & $121(53.5)$ & 0.597 \\
\hline Heart failure $^{b}$ & $58(11.5)$ & $21(9.3)$ & 0.377 \\
\hline Coronary artery disease & $61(12.1)$ & 39 (17.3) & 0.060 \\
\hline Cancer & 117 (23.2) & $75(33.2)$ & 0.004 \\
\hline Acute renal failure $^{c}$ & $124(24.6)$ & $76(33.6)$ & 0.011 \\
\hline $\operatorname{ARDS}^{d}$ & $43(8.5)$ & $28(12.4)$ & 0.102 \\
\hline \multicolumn{4}{|l|}{ Laboratory parameter } \\
\hline $\operatorname{WBC}\left(10^{3} / \mu l\right)$ & $14.2 \pm 11.2$ & $15.1 \pm 11.8$ & 0.888 \\
\hline Hct $(\%)$ & $29.7 \pm 6.5$ & $28.0 \pm 7.0$ & 0.002 \\
\hline Platelet $\left(10^{3} / \mathrm{mm}^{3}\right)$ & $181.8 \pm 116.9$ & $135.3 \pm 118.5$ & $<0.001$ \\
\hline Creatinine (mg/dl) & $2.7 \pm 16.7$ & $1.9 \pm 1.7$ & 0.457 \\
\hline Total bilirubin (mg/dl) & $1.2 \pm 2.6$ & $1.8 \pm 2.7$ & 0.007 \\
\hline Albumin (g/dl) & $2.6 \pm 0.5$ & $2.4 \pm 0.6$ & $<0.001$ \\
\hline $\mathrm{CRP}$ (mg/dl) & $114.5 \pm 106.7$ & $135.3 \pm 106.9$ & 0.015 \\
\hline Procalcitonin (ng/ml) & $12.5 \pm 28.9$ & $13.2 \pm 30.1$ & 0.781 \\
\hline Bilirubin/albumin ratio & $0.4 \pm 0.9$ & $0.7 \pm 1.0$ & $<0.001$ \\
\hline
\end{tabular}

Values are presented as mean \pm standard deviation or number (\%). ICU, intensive care unit; BMI: body mass index; APACHE: Acute Physiology and Chronic Health Evaluation; SOFA: Sequential Organ Failure Assessment; SAPS: Simplified Acute Physiology Score; ARDS: acute respiratory distress syndrome; WBC: white blood cell; Hct: hematocrit; CRP: C-reactive protein.

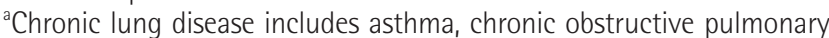
disease, and structural lung diseases, such as bronchiectasis and interstitial lung disease: ${ }^{b} \mathrm{~A}$ complex clinical syndrome that results from any structural or functional impairment of ventricular filling or ejection of blood; 'An increase over 1.5 times or $0.3 \mathrm{mg} / \mathrm{dl}$ in serum creatinine within 7 days compared to baseline or a urine output less than $0.5 \mathrm{ml} / \mathrm{kg} / \mathrm{hr}$ during 6 to 12 hours according to the Kidney Disease: Improving Global Outcomes guidelines; ' ${ }^{\top}$ The rapid onset of hypoxemia and pulmonary edema with diffuse bilateral pulmonary infiltrates from pulmonary or non-pulmonary disease when the $\mathrm{PaO}_{2} / \mathrm{FiO}_{2}$ ratio was less than $300 \mathrm{~mm}$ $\mathrm{Hg}$ at the time of ICU admission.

\section{RESULTS}

Among a total of 867 patients who admitted to the medical ICU at our university hospital in Korea, 136 patients who had the acute toxic or alcoholic hepatitis, chronic hepatitis, liver cirrhosis, cholecystitis with or without cholangitis, and those who underwent liver, biliary tract, or pancreatic surgery were excluded. Therefore, 731 patients were finally enrolled in our study; 448 (61.3\%) were men and $283(38.7 \%)$ were women (Figure 1). The mean age was $66.4 \pm 14.7$ years. The most common underlying disease was hypertension $(n=402,55.0 \%)$, followed by diabetes mellitus ( $n=262,35.8 \%)$, solid cancers $(n=192,26.3 \%)$, and hematologic malignancies $(\mathrm{n}=45,6.2 \%)$. The mean APACHE II score, SOFA score, and SAPSII were $23.5 \pm 10.5,8.6 \pm 4.1$, and $41.8 \pm 17.2$, respectively. In total, 470 patients $(64.3 \%)$ were intubated for acute respiratory failure. The overall 28-day survival of MICU patients was $69.1 \%(n=505)$; these patients were defined as survivors, and the remaining patients $(\mathrm{n}=226,30.9 \%)$ were defined as non-survivors. Table 1. showed the comparison of baseline characteristics, clinical, and laboratory parameters between survival and non-survivors. On univariate analysis, age $(\mathrm{P}=0.043)$ and APACHE II score $(\mathrm{P}<0.001)$ were significantly higher in non-survivors. And, the incidence of cancer $(\mathrm{P}=0.004)$ and acute renal failure $(\mathrm{P}=0.011)$ was higher in non-survivors. Serum hematocrit $(\mathrm{P}=0.002)$, platelet $(\mathrm{P}<0.001)$, and albumin $(\mathrm{P}<0.001)$ were significantly lower in non-survivors than in survivors. Total bilirubin $(\mathrm{P}=0.007)$, $\mathrm{C}$-reactive protein $(\mathrm{P}=0.015)$, and bilirubin/albumin ratio $(\mathrm{P}<0.001)$ were positively associated with mortality of ICU patients (Table 1 ).

The $\mathrm{B} / \mathrm{A}$ ratio was significantly associated with 28 -day mortality in patients with admission in ICU on multivariate analysis adjusted for age, sex, BMI, underlying disease, and APACHE II

Table 2. Multivariate analysis to predict 28-day mortality in ICU

\begin{tabular}{lccc}
\hline Variable & OR & $95 \% \mathrm{Cl}$ & P-value \\
\hline Age & 1.00 & $0.99-1.01$ & 0.489 \\
Sex & & & \\
$\quad$ Male & Reference & Reference & Reference \\
$\quad$ Female & 1.20 & $0.84-1.68$ & 0.309 \\
BMI & 0.98 & $0.95-1.00$ & 0.202 \\
APACHE II score & 1.06 & $1.04-1.07$ & $<0.001$ \\
Underlying disease & & & \\
Cancer & 1.70 & $1.18-2.43$ & 0.004 \\
Bilirubin/albumin & 1.27 & $1.07-1.51$ & 0.006 \\
\hline
\end{tabular}

ICU: intensive care unit; OR: odds ratio; $\mathrm{Cl}$ : confidence interval; BMI: body mass index; APACHE: Acute Physiology and Chronic Health Evaluation. 


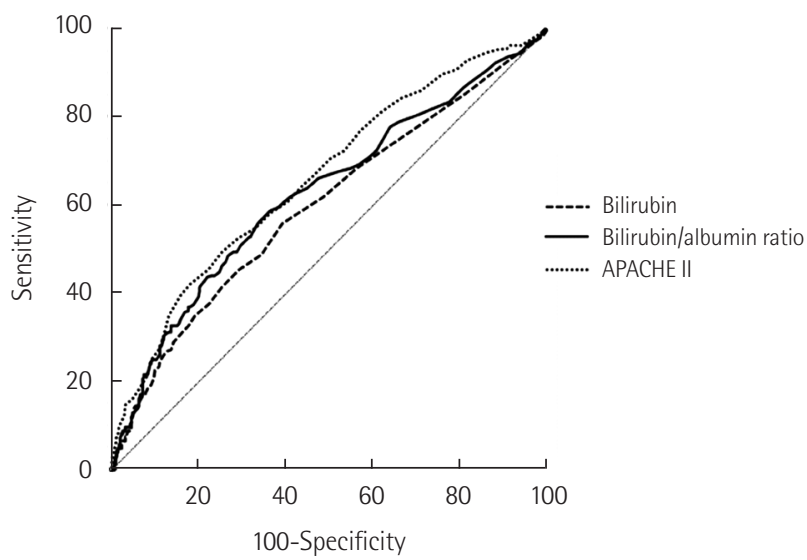

Figure 2. Receiver operating characteristic (ROC) curve of serum bilirubin, bilirubin/albumin ratio, and Acute Physiology and Chronic Health Evaluation (APACHE) II score curves as predictors of 28day mortality. The area under curve for the bilirubin/albumin ratio is not significantly inferior to APACHE II score $(\mathrm{P}=0.282)$.

Table 3. Cox proportional-hazard regression analysis

\begin{tabular}{|c|c|c|c|}
\hline Variable & $H R$ & $95 \% \mathrm{Cl}$ & P-value \\
\hline Age & 0.99 & $0.98-1.01$ & 0.632 \\
\hline \multicolumn{4}{|l|}{ Sex } \\
\hline Male & Reference & Reference & Reference \\
\hline Female & 1.22 & $0.93-1.60$ & 0.150 \\
\hline BMI & 0.98 & $0.96-1.01$ & 0.147 \\
\hline APACHE II score & 1.05 & $1.03-1.06$ & $<0.001$ \\
\hline \multicolumn{4}{|l|}{ Underlying disease } \\
\hline Cancer & 1.41 & $1.07-1.86$ & 0.015 \\
\hline \multicolumn{4}{|l|}{ Bilirubin/albumin } \\
\hline $\operatorname{Low}(\leq 0.31)$ & Reference & Reference & Reference \\
\hline High (>0.31) & 1.65 & $1.23-2.20$ & 0.001 \\
\hline
\end{tabular}

HR: hazard ratio; $\mathrm{Cl}$ : confidence interval; BMI: body mass index; APACHE: Acute Physiology and Chronic Health Evaluation.

score (OR, 1.27; 95\% CI, 1.07 to 1.51, $\mathrm{P}=0.006$ ) (Table 2). Figure 2 shows the ROC curves for bilirubin, $\mathrm{B} / \mathrm{A}$ ratio and APACHE II score, and the AUC was $0.599,0.628$ and 0.666 for mortality in ICU patients. The ROC curves between $\mathrm{B} / \mathrm{A}$ ratio and APACHE II score were not significantly different $(\mathrm{P}=0.282)$. The sensitivity and specificity for 28 -day mortality were $58.4 \%$ and $64.2 \%$ at a B/A ratio cutoff of 0.31 .

The Table 3 and Figure 3 represent the results of survival analysis. A higher B/A ratio ( $>0.31$ ) was significantly correlated with lower 28-day mortality rates on Kaplan-Meier survival analysis $(\mathrm{P}<0.001)$ (Figure 3). The effect of age, sex, BMI, APACHE II score, underlying disease, and $\mathrm{B} / \mathrm{A}$ ratio for 28-day mortality

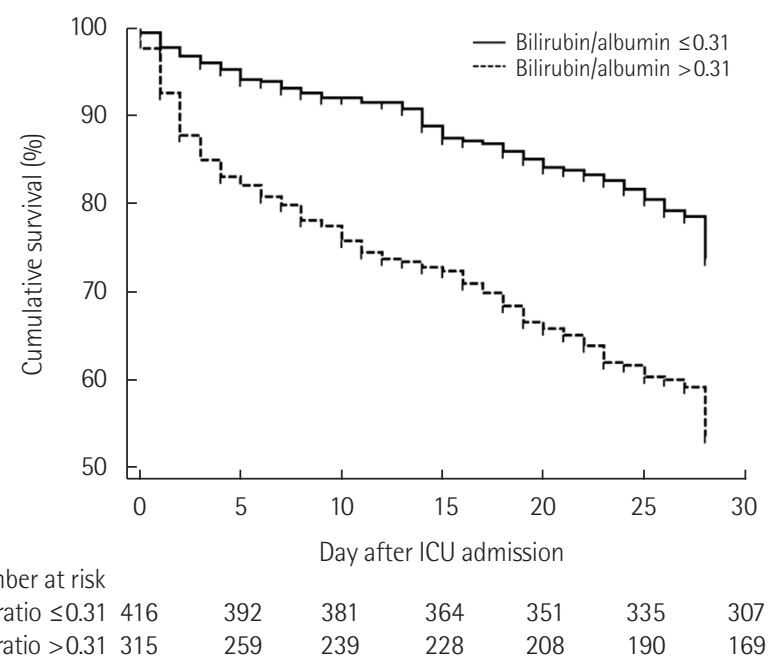

Figure 3. Kaplan-Meier analysis of time to death in intensive care unit (ICU) patients according to bilirubin to albumin (B/A) ratio $\leq 0.31$ or $>0.31(\mathrm{P}<0.001)$.

was investigated using the Cox proportional hazard model (Table 3). For Kaplan-Meier survival analysis, patients were separated into two groups according to $\mathrm{B} / \mathrm{A}$ ratios at a cutoff value of 0.31 . The relative risk of mortality was significantly associated with $\mathrm{B} / \mathrm{A}$ ratio (hazard ratio [HR], 1.65; 95\% confidence interval [CI], 1.23 to $2.20 ; \mathrm{P}=0.001)$.

\section{DISCUSSION}

In this study, we found that initial $\mathrm{B} / \mathrm{A}$ ratio was significantly associated with higher 28-day mortality in ICU patients. Based on ROC curve analysis, a B/A ratio cutoff values of 0.31 was identified for predicting the 28-day mortality. The large number of patients in our study could strength to the relevance of the results. These results strengthen our knowledge about the role of B/A ratio as a predictive factor for poor outcomes in critically ill patients.

Several previous reports about the mechanisms underlying liver dysfunction and jaundice in critical illnesses have been published. The liver is involved in host defense mechanisms and tissue repair facilitated by cross-talk and interactions among hepatic cell including hepatocytes, Kupffer cells, and endothelial sinusoidal cells in patients with sepsis and other critical illnesses [1]. However, over-activation of the systemic response, hypoxemia, and unexpected side effects of treatment could result in hepatic dysfunction [2]. The incidence of hepatic dysfunction resulting to abnormal liver test was reported in 10-30\% of ICU patients $[5,6,16]$.

Liver function test abnormalities were commonly appeared 
in critically ill patients from the two major pathogenesis: hypoxic hepatitis and cholestasis caused by critical illness [17]. An initial and disruptive increase of the liver lysis enzymes, including ALT and AST, in hypoxic hepatitis or shock liver was reflected diffuse hepatic injury as a result from the failure of oxygen delivery and ischemia in the liver from severe shock. [18,19]. According to a previous report, jaundice could develop in one-third of patients with hypoxic hepatitis [20]. Cholestasis followed by critical illness is more frequently manifested in the ICU and is characterized by mild abnormalities in hepatic function test with mainly cholestatic features. Hypoxic hepatitis is apparently associated with severe shock and liver hypoxia. However, it seems that cholestasis caused by critical illness are represented by cellular alterations from inflammation leading to a transient bile acid accumulation in the liver and systemic circulation, unlike hypoxic hepatitis is associated with severe shock and liver hypoxia $[6,20,21]$.

Serum bilirubin concentration is usually conducted to evaluated the liver function in ICU patients. Increased liver lysis enzymes are common in all cases that altered tissue perfusion occur. In addition, coagulation tests including prothrombin time and partial thromboplatin time are easily affected in many diseases with coagulopathy, such as disseminated intravascular coagulation. On the other hand, serum bilirubin is easily measured and obtained. Therefore, the bilirubin levels were included in already used organ dysfunction scoring system, such as SOFA [22], SAPS [9], and Logistic Organ Dysfunction Score [23].

As a marker of liver dysfunction, elevation of bilirubin level has been considered to be directly related to prognosis in critically ill patients. Brienza et al. [5] found that the prevalence of hyperbilirubinemia with definition of that value more than 2 $\mathrm{mg} / \mathrm{dL}$ was $30 \%$ in critically ill patient. In their study, sepsis, severe septic shock, mechanical ventilation with Positive End Expiratory Pressure, and major surgery were risk factors for hyperbilirubinemia [5]. Recently, Pierrakos et al. [7] reported that hyperbilirubinemia without primary cause could be an independent risk factor of mortality in ICU patient. In that study, the relationship between bilirubin and mortality presented a linear correlation from the value of 1 to $6 \mathrm{mg} / \mathrm{dl}$. [7]. In our study, serum bilirubin level at ICU admission was a predictor for 28-day mortality. The results of our study were reliable to previous reports. In fact, in general, not only a marker of liver dysfunction, bilirubin can itself affect results. In the previous reports, the bactericidal characters of neutrophils could be impaired by antioxidative effects of bilirubin [24]. Furthermore, bilirubin can have nephrotoxic [25] and neurotoxic [26] effects. In addition, it can inhibit induced nitric oxide synthase [27] and exert platelet anti-aggregation effects, possible by inhibiting platelet activation induced from collagen [28]. Because we analyzed bilirubin levels relatively early on (at ICU admission), the toxicity of bilirubin itself may have affected mortality in this study.

In addition, hypoalbuminemia is frequently appeared in critically ill patients, and lower serum albumin was associated with the higher mortality in acute ill patients $[4,10]$. In prior metaanalysis of 90 cohort studies, lower serum albumin level was a dose-dependent prognostic factor for poor outcome including mortality, morbidity, and prolonged stay in ICU and hospital [4]. The relationship between hypoalbuminemia and worse outcome seemed to be independent from inflammation and nutritional status in this study [4]. In a prospective cohort study conducted in a single center ICU, APACHE II and albumin were associated with poor outcome and mortality independently in critically ill patients with severe sepsis or septic shock [10]. Because of its value as an prognostic factor for mortality, serum albumin level was included to one of the component parameters in APACHE III score [11].

In this study, we analyzed B/A ratio for predictor of mortality. In the previous reports, serum B/A ratio was expected as a predictor of bilirubin encephalopathy and neurologic dysfunction in neonates $[12,29,30]$. Free bilirubin, that is not bound to albumin, can pass by the blood-brain-barrier and appear a neurotoxicity $[12,29,30]$. The $\mathrm{B} / \mathrm{A}$ ratio, which can be a substitute for free bilirubin, is considered to be a good additional parameter for total serum bilirubin, indicating an higher risk of bilirubininduced neurotoxicity in preterm infants $[12,29,30]$. However, the role of $\mathrm{B} / \mathrm{A}$ ratio as a predictor for mortality in critically ill patient has not been evaluated before. In this study, the AUC of the $\mathrm{B} / \mathrm{A}$ ratio was significantly higher than that of bilirubin and was not inferior to that of APACHE II score. A higher B/A ratio at ICU admission was associated with unfavorable outcomes in critically ill patients.

This is the first study to investigate the role of $\mathrm{B} / \mathrm{A}$ ratio in predicting the prognosis of critically ill adult patients in the ICU. Although there are several scoring systems for predicting the mortality in patients with critically illness, our study result of B/A ratio is valuable in that it can be measured simply and easy to use everywhere. Furthermore, the predictive quality of the B/A ratio is not inferior to APACHE II score.

Nevertheless, our study has some limitations. First, this study result may be difficult to generalize because of a single-center retrospective observational study. Second, we were unable to measure urine output before admission to the ICU. Third, we could not assess changes in bilirubin, albumin, and B/A ratios, because serum albumin and bilirubin level were only collected 
at first day of ICU admission. So, it is needed in the future to further studies which investigate about the changes in those markers.

The results of this study had showed that a higher B/A ratio might be related to higher mortality and worse outcome in critically ill patients. Hence, clinicians can use the initial measurement of B/A ratio to predict the prognosis of ICU patients. Multicenter studies with large samples need to be conducted for validation of these results and presenting the serum $\mathrm{B} / \mathrm{A}$ ratio as a valuable marker for prediction of prognosis in critically ill patients.

\section{CONFLICT OF INTEREST}

No potential conflict of interest relevant to this article was reported.

\section{ORCID}

$\begin{array}{ll}\text { Ji Soo Choi } & \text { https://orcid.org/0000-0003-2716-6775 } \\ \text { Kyung Soo Chung } & \text { https://orcid.org/0000-0003-1604-8730 } \\ \text { Eun Hye Lee } & \text { https://orcid.org/0000-0003-2570-3442 } \\ \text { Su Hwan Lee } & \text { https://orcid.org/0000-0002-3487-2574 } \\ \text { Sang Hoon Lee } & \text { https://orcid.org/0000-0002-7706-5318 } \\ \text { Song Yee Kim } & \text { https://orcid.org/0000-0001-8627-486X } \\ \text { Ji Ye Jung } & \text { https://orcid.org/0000-0003-1589-4142 } \\ \text { Young Ae Kang } & \text { https://orcid.org/0000-0002-7783-5271 } \\ \text { Moo Suk Park } & \text { https://orcid.org/0000-0003-0820-7615 } \\ \text { Young Sam Kim } & \text { https://orcid.org/0000-0001-9656-8482 } \\ \text { Joon Chang } & \text { https://orcid.org/0000-0003-4542-6841 } \\ \text { Ah Young Leem } & \text { https://orcid.org/0000-0001-5165-3704 }\end{array}$

\section{AUTHOR CONTRIBUTIONS}

Conceptualization: AYL, JSC. Data curation: AYL, KSC, JSC. Formal analysis: AYL, JSC. Methodology: AYL, JSC. Writing original draft: AYL, JSC. Writing - review \& editing: all authors.

\section{REFERENCES}

1. Dhainaut JF, Marin N, Mignon A, Vinsonneau C. Hepatic response to sepsis: interaction between coagulation and inflammatory processes. Crit Care Med 2001;29(7 Suppl):S42-7.

2. Jenniskens M, Langouche L, Van den Berghe G. Cholestatic alterations in the critically ill: some new light on an old problem. Chest 2018;153:733-43.

3. Hawker F. Liver dysfunction in critical illness. Anaesth Inten- sive Care 1991;19:165-81.

4. Vincent JL, Dubois MJ, Navickis RJ, Wilkes MM. Hypoalbuminemia in acute illness: is there a rationale for intervention? A meta-analysis of cohort studies and controlled trials. Ann Surg 2003;237:319-34.

5. Brienza N, Dalfino L, Cinnella G, Diele C, Bruno F, Fiore T. Jaundice in critical illness: promoting factors of a concealed reality. Intensive Care Med 2006;32:267-74.

6. Kramer L, Jordan B, Druml W, Bauer P, Metnitz PG; Austrian Epidemiologic Study on Intensive Care, ASDI Study Group. Incidence and prognosis of early hepatic dysfunction in critically ill patients: a prospective multicenter study. Crit Care Med 2007;35:1099-104.

7. Pierrakos C, Velissaris D, Felleiter P, Antonelli M, Vanhems P, Sakr Y, et al. Increased mortality in critically ill patients with mild or moderate hyperbilirubinemia. J Crit Care 2017;40:3135 .

8. Jones AE, Trzeciak S, Kline JA. The Sequential Organ Failure Assessment score for predicting outcome in patients with severe sepsis and evidence of hypoperfusion at the time of emergency department presentation. Crit Care Med 2009;37:164954.

9. Le Gall JR, Lemeshow S, Saulnier F. A new Simplified Acute Physiology Score (SAPS II) based on a European/North American multicenter study. JAMA 1993;270:2957-63.

10. Artero A, Zaragoza R, Camarena JJ, Sancho S, Gonzalez R, Nogueira JM. Prognostic factors of mortality in patients with community-acquired bloodstream infection with severe sepsis and septic shock. J Crit Care 2010;25:276-81.

11. Knaus WA, Wagner DP, Draper EA, Zimmerman JE, Bergner M, Bastos PG, et al. The APACHE III prognostic system. Risk prediction of hospital mortality for critically ill hospitalized adults. Chest 1991;100:1619-36.

12. Ardakani SB, Dana VG, Ziaee V, Ashtiani MT, Djavid GE, Alijani M. Bilirubin/albumin ratio for predicting acute bilirubininduced neurologic dysfunction. Iran J Pediatr 2011;21:28-32.

13. Hulzebos CV, van Imhoff DE, Bos AF, Ahlfors CE, Verkade HJ, Dijk PH. Usefulness of the bilirubin/albumin ratio for predicting bilirubin-induced neurotoxicity in premature infants. Arch Dis Child Fetal Neonatal Ed 2008;93:F384-8.

14. Zhang Z. Model building strategy for logistic regression: purposeful selection. Ann Transl Med 2016;4:111.

15. Zhang Z. Residuals and regression diagnostics: focusing on logistic regression. Ann Transl Med 2016;4:195.

16. Kortgen A, Paxian M, Werth M, Recknagel P, Rauchfuss F, Lupp A, et al. Prospective assessment of hepatic function and mechanisms of dysfunction in the critically ill. Shock 2009;32:358- 
65.

17. Horvatits T, Trauner M, Fuhrmann V. Hypoxic liver injury and cholestasis in critically ill patients. Curr Opin Crit Care 2013; 19:128-32.

18. Fuhrmann V, Kneidinger N, Herkner H, Heinz G, Nikfardjam M, Bojic A, et al. Hypoxic hepatitis: underlying conditions and risk factors for mortality in critically ill patients. Intensive Care Med 2009;35:1397-405.

19. Fuhrmann V, Kneidinger N, Herkner H, Heinz G, Nikfardjam M, Bojic A, et al. Impact of hypoxic hepatitis on mortality in the intensive care unit. Intensive Care Med 2011;37:1302-10.

20. Jager B, Drolz A, Michl B, Schellongowski P, Bojic A, Nikfardjam $\mathrm{M}$, et al. Jaundice increases the rate of complications and one-year mortality in patients with hypoxic hepatitis. Hepatology 2012;56:2297-304.

21. Mesotten D, Wauters J, Van den Berghe G, Wouters PJ, Milants I, Wilmer A. The effect of strict blood glucose control on biliary sludge and cholestasis in critically ill patients. J Clin Endocrinol Metab 2009;94:2345-52.

22. Vincent JL, Moreno R, Takala J, Willatts S, De Mendonca A, Bruining $\mathrm{H}$, et al. The SOFA (Sepsis-related Organ Failure Assessment) score to describe organ dysfunction/failure. On behalf of the Working Group on Sepsis-Related Problems of the European Society of Intensive Care Medicine. Intensive Care Med 1996;22:707-10.

23. Le Gall JR, Klar J, Lemeshow S, Saulnier F, Alberti C, Artigas A, et al. The Logistic Organ Dysfunction system. A new way to assess organ dysfunction in the intensive care unit. ICU Scor- ing Group. JAMA 1996;276:802-10.

24. Arai T, Yoshikai Y, Kamiya J, Nagino M, Uesaka K, Yuasa N, et al. Bilirubin impairs bactericidal activity of neutrophils through an antioxidant mechanism in vitro. J Surg Res 2001;96:107-13.

25. Uslu A, Tasli FA, Nart A, Postaci H, Aykas A, Bati H, et al. Human kidney histopathology in acute obstructive jaundice: a prospective study. Eur J Gastroenterol Hepatol 2010;22:145865.

26. Dubois MJ, Bergeron N, Dumont M, Dial S, Skrobik Y. Delirium in an intensive care unit: a study of risk factors. Intensive Care Med 2001;27:1297-304.

27. Lanone S, Bloc S, Foresti R, Almolki A, Taille C, Callebert J, et al. Bilirubin decreases nos 2 expression via inhibition of NAD(P)H oxidase: implications for protection against endotoxic shock in rats. FASEB J 2005;19:1890-2.

28. Kundur AR, Bulmer AC, Singh I. Unconjugated bilirubin inhibits collagen induced platelet activation. Platelets 2014;25: 45-50.

29. Hulzebos CV, Dijk PH, van Imhoff DE, Bos AF, Lopriore E, Offringa $\mathrm{M}$, et al. The bilirubin albumin ratio in the management of hyperbilirubinemia in preterm infants to improve neurodevelopmental outcome: a randomized controlled trial. BARTrial. PLoS One 2014;9:e99466.

30. Iskander I, Gamaleldin R, El Houchi S, El Shenawy A, Seoud I, El Gharbawi N, et al. Serum bilirubin and bilirubin/albumin ratio as predictors of bilirubin encephalopathy. Pediatrics 2014; 134:e1330-9. 